\title{
Study of Perinatal Outcome of Severe Late onset Intrauterine Growth Restriction using Biophysical and Doppler Fetal Parameters \\ Medhat Essam Eldeen Helmy ${ }^{1}$, Mohamed Ehab Ali Salit ${ }^{2}$, Shaimaa Abdel-hamid Hassanein ${ }^{3}$, Ahmed Sabry Fouad El-hamouly ${ }^{1}$, Mohamed ELsibai Anter*1,
}

Departments of ${ }^{1}$ Obstetrics \& Gynecology, ${ }^{3}$ Diagnostic Radiology, Faculty of Medicine, Menoufia University. Department of ${ }^{2}$ Obstetrics and Gynecology, Faculty of Medicine, Cairo University *Corresponding author: Mohamed ELsibai Anter, Mobile: (+20) 01009737067, E-Mail: mohamedsibai681@yahoo.com

\begin{abstract}
Background: Intrauterine growth restriction is one of the most common causes of perinatal morbidity and mortality worldwide. Strong evidence about the optimum method of surveillance is needed.

Objective: to determine the relationship between biophysical fetal parameters and arterial and venous Doppler parameters in fetuses with severe intrauterine growth restriction (IUGR).

Patients and Methods: This is a Prospective cohort study was conducted at Menoufia University Hospital during the period from March 2019 to September 2020. Fourty two IUGR fetuses with elevated Umbilical Artery (UA) Doppler had follow up with Doppler (UA, middle cerebral artery and ductus venosus) and Biophysical profile (BPP). Patients were stratified into three groups: G1: abnormal UA alone. G2: brain sparing effect. G3: abnormal DV Doppler. Our main primary outcome was neonatal intensive care unit (NICU) admission while secondary outcomes were APGAR score, Birth weight, EFW and stillbirth rate.

Results UAEDV was present in 29 fetuses (69\%), Absent/Reversed (AREDV) in 13 (31\%). Brain sparing was observed in 18 fetuses (42.85\%). Abnormal DV Doppler was noticed in 5 fetuses (11.9\%). The BPP was > 6 in 28 fetuses (66.6\%). Abnormal doppler (UA, MCA, DV) and abnormal BPP were significantly associated with increased NICU admissions, Still birth rates, lower APGAR scores, EFW and Birth weight. Multivariate regression analysis was performed, and it was found that abnormal MCA was the most associated parameter with NICU admission. Conclusion: Fetal Doppler (UA, MCA, DV) and BPP are complementary antenatal modalities. Therefore, pursuit of a combined testing approach has merit.
\end{abstract}

Keywords: Biophysical profile, Doppler, Fetal monitoring, Growth restriction.

\section{INTRODUCTION}

Intrauterine growth restriction (IUGR) undoubtedly is one of the most common causes of perinatal morbidity and mortality worldwide. Historically, IUGR has been defined as fetuses whose estimated fetal weight falls below the 10th percentile for gestational age (1). Doppler ultrasonography and biophysical profile scoring (BPP) are the principal surveillance tools in pregnancies complicated by placental vascular insufficiency and IUGR ${ }^{(2)}$. These antenatal testing modalities aim to detect fetal compromise by evaluating fetal manifestations of altered oxygenation and metabolic status ${ }^{(3)}$. As placental blood flow resistance worsens, changes in cerebral blood flow resistance are observed in the compensated state. Finally, deteriorations in venous Doppler parameters are observed that reflect alterations in cardiac forward function in response to advanced placental insufficiency (4). Multi-vessel Doppler examination is able to accurately depict this progression in IUGR fetuses. Dynamic fetal variables (movement, tone, breathing, heart rate reactivity and amniotic fluid volume) utilized for BPP remain normal longer in the progression of IUGR fetuses ${ }^{(5)}$. With progressive fetal compromise reduction of global fetal activity is followed by a decline in fetal heart rate reactivity and fetal breathing movement and amniotic fluid volume ${ }^{(6)}$. Finally, fetal tone and movement are lost in response to worsening acidemia ${ }^{(7)}$.
Previous studies clearly show that cardiovascular and biophysical responses in IUGR fetuses both relate to fetal status. To determine which combination of antenatal tests provides the best reflection of fetal status, it is first necessary to assess the relationship between test results in these principal modalities ${ }^{(\mathbf{8})}$.

If deteriorations in both Doppler and biophysical parameters are closely related, then test results should be concordant in the majority of cases.

On the other hand, if deterioration in cardiovascular and behavioral status occurs independently of each other a certain degree of disagreement between the test results is to be expected. It was the aim of this study to determine the distribution and relationship of Doppler and BPP test results in a population of IUGR fetuses with placental insufficiency (9).

\section{PATIENTS AND METHODS}

Study Setting: This is a Prospective cohort study was conducted at Obstetrics and Gynecology Department, Menoufia University Hospital during the period from March 2019 to September 2020.

Eligibility criteria: The participants were chosen according to appropriate inclusion and exclusion criteria. Fourty two patient fulfilled the inclusion criteria. The inclusion criteria was; Normal fetal 
anatomy, Sure of date, Fetal abdominal circumference $<10$ th percentile for gestational age and Expected Fetal Weight < 10th percentile for GA by reference values and Umbilical artery Doppler pulsatility index (PI) more than 2 standard deviations (SDs) above the gestational mean by local reference. Exclusion criteria was; unknown date or Diagnosed congenital or chromosomal anomalies.

Data collection: All participants were received at department of Obstetrics and Gynecology, Menoufia University hospitals where they provided full personal and medical history and underwent abdominal and general examination with detailed ultrasound examination and BPP. Each participant underwent follow up with Doppler (UA, MCA, DV) and BPP. Gestational Age was determined according to date of last menstrual period (LMP) or 1st trimester crown rump length (CRL) by ultrasound. Body mass index (BMI) was calculated (weight in $\mathrm{kg} /$ height in $\mathrm{m} 2$ ).

Settings and procedures: GE Voluson Ultrasound Machine was used for detailed obstetric ultrasound and Doppler examination. Pulsed-wave Doppler measurements of the UA, MCA and DV were obtained using the pulsatility index (PI) to quantify arterial
Doppler waveforms and the PIV for veins or the systolic a-wave to quantify the DV waveform.

Biophysical profile scoring: The five-component BPP was performed during the fetal assessment. Fetal breathing movements sustained for $30 \mathrm{~s}$ or more, presence of at least three body/limb movements and at least one vigorous flexion/extension episode of the limbs each yielded 2 points. A maximum vertical amniotic fluid pocket above $2 \mathrm{~cm}$ yielded 2 points. Non stress test (NST) after 30 weeks' gestation, two 15-beat accelerations, each sustained for $15 \mathrm{~s}$. Two points were given if these criteria were met.No points were given for any components if these cut-offs were not met in a 30min period.

\section{Procedure of Doppler evaluation performed:}

Umbilical Artery: A loop of umbilical cord close to the placenta was located. The segment of umbilical cord elongated so that the two umbilical arteries and one umbilical vein would be distinguished. Angle of insonation adjusted to less than 60 degrees. An optimum Doppler signal obtained and the pulsatility index measured. In the UA Doppler: absence or reversal of end diastolic velocities (UA-A/REDV) was noted. All fetuses had an elevated UA-PI (> 2 SD) as part of their entry criteria. (Figure 1).

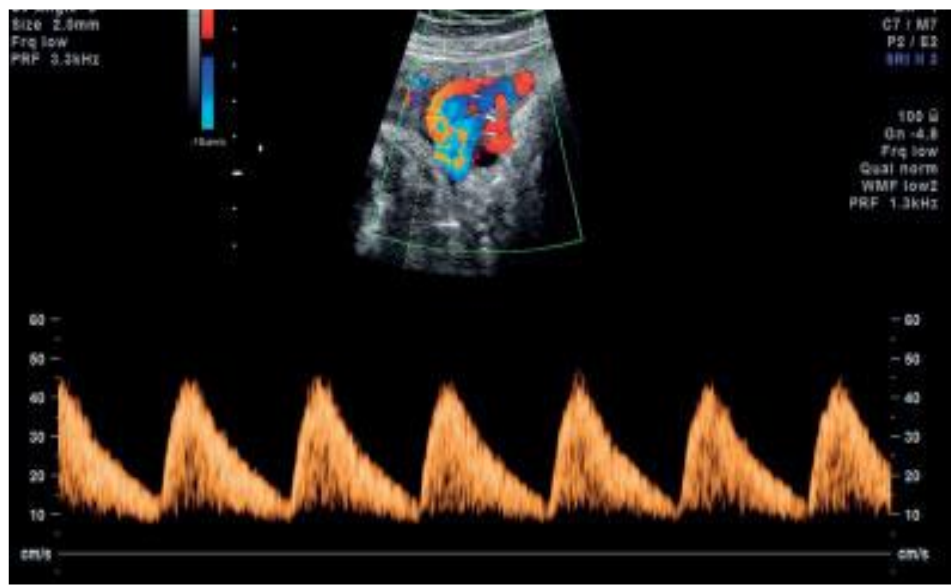

Figure (1): Showing UA Doppler PI more than 2 SD from mean.

Fetal Middle Cerebral Artery: Section of fetal head used for BPD measurement obtained and then the transducer angled caudally till the middle cerebral artery is seen. Sample volume size and angle of insonation adjusted after placing the cursor over the artery and appropriate signals obtained. An MCA-PI greater than 2 standard deviations (SD) below the mean for gestational age was considered as evidence of 'brain sparing'. (Figure $2 \mathrm{a}+\mathrm{b}$ )

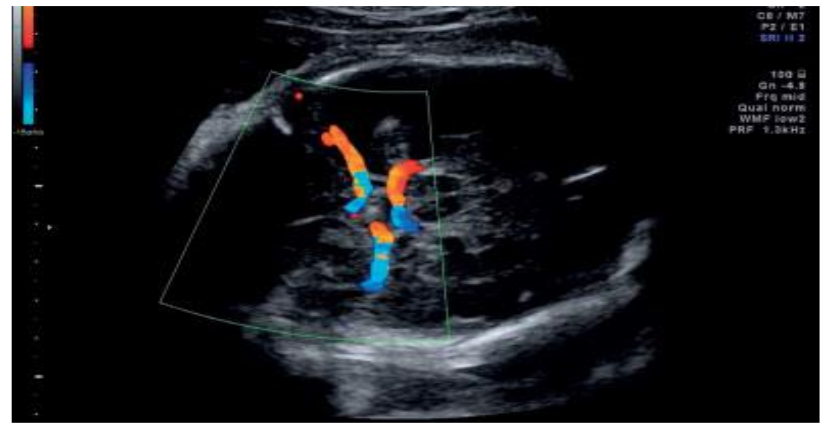

(a)

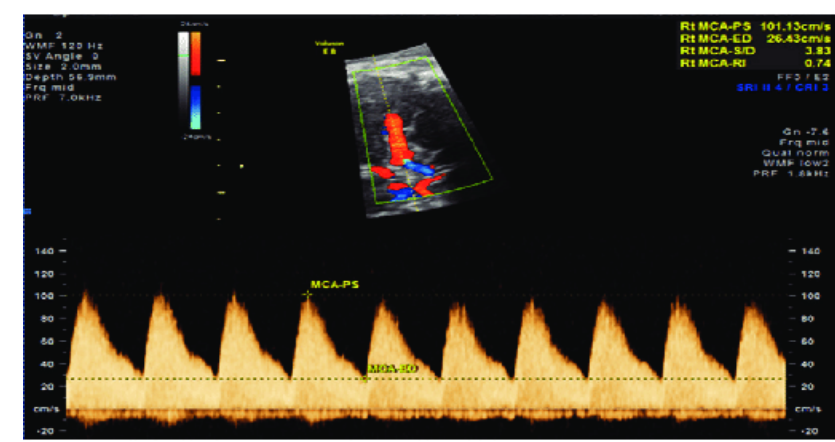

(b)

Figure (2): $(a+b)$ showing Doppler wave of MCA. 
Fetal Ductus Venosus: A transverse section of the fetal abdomen obtained with the transducer angled slightly cephalad, color flow switched on and the aliasing signal in the DV identified where it connects the umbilical vein to the IVC. The sample volume and the angle of waveform obtained. The PI was then be measured. The DV Doppler PIV index elevation greater than 2 SD above the mean for gestational age was considered elevated. (Figure 3)

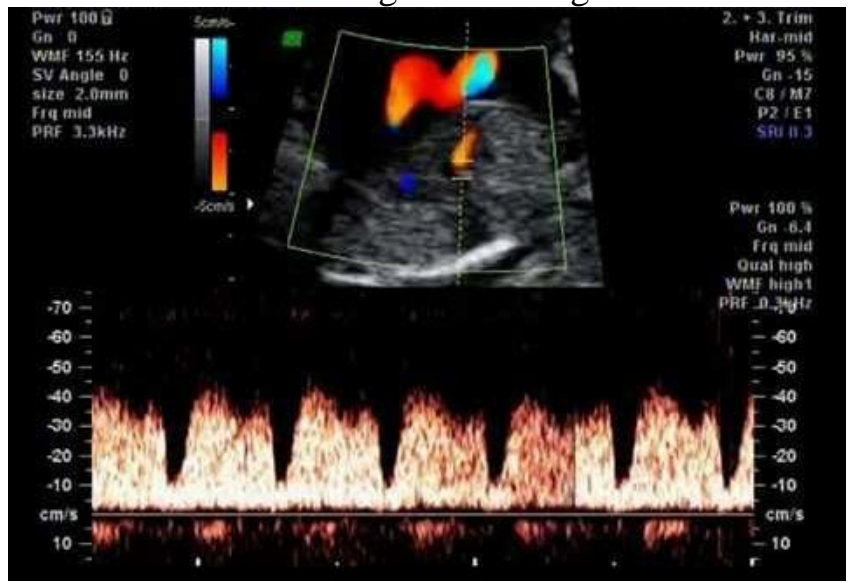

Figure (3): High DV Doppler wave

Primary outcome: NICU Admission. Secondary outcomes: APGAR score at 1,5 minutes, Birth weight, EFW and stillbirth rate.

\section{Ethical approval:}

The study was approved by local ethical committee. Women who agreed to participate gave their signed informed consent after explanation of the study benefits and hazards. All procedures were carried out in accordance with the ethical standards of the institutional and/or national research committee and with the 1964 Declaration of Helsinki and its later amendments or comparable ethical standards.

Sample Size: calculated according to a study done by Baschat ${ }^{(10)}$, to achieve $90 \%$ power to detect this difference with a significant level of $5 \%$, it is estimated that 42 subjects with established late onset growth restricted fetuses after 30 weeks were required.

Statistical Analysis: Results were statistically analyzed by SPSS version 22(SPSS Inc., Chikago, IL, USA). No-paired t test was used for parametric data. Mann-Whitney was used for non-parametric data. Chi-Squared $(\chi 2)$ and Fisher's exact tests were used for qualitative variables. Spearman correlation tests were used for detecting the strength and direction of association between variables. Binary logestic regression was used to assess the predictors of an outcome. $\mathrm{P}$ value $<0.05$ is considered significant.

\section{RESULTS}

Table (1): General characteristics of the studied group:

\begin{tabular}{||l|c|c|}
\hline \multirow{2}{*}{ General characteristics } & \multicolumn{2}{|c|}{ Participants (n=42) } \\
\cline { 2 - 3 } & Mean \pm SD & Range \\
\hline Maternal Age (Y) & $27.59 \pm 6.03$ & $19.0-41.0$ \\
\hline Body Mass Index (kg/m2) & $28.93 \pm 6.84$ & $16.5-41.0$ \\
\hline & no & $\%$ \\
\hline Parity & 22 & \\
Nulliparous & 20 & 52.4 \\
Multiparous & & 47.6 \\
\hline Comorbidity & 13 & \\
Yes (PET, anemia, bronchial asthma, DM, Epilepsy, SLE). & 29 & 31.0 \\
No & 4 & 69.0 \\
\hline Smoking & 8 & 9.5 \\
\hline Past history of IUGR & & 19.0 \\
\hline Mode of Delivery & 31 & \\
Caeserian Section & 11 & 73.8 \\
Normal Vaginal Delivery & Mean \pm SD & 26.2 \\
\hline Gestational age & $32.52 \pm 2.02$ & $32.0(31.0-39.0)$ \\
\hline At study entry (week) & $35.65 \pm 1.94$ & $36.0(32.0-39.0)$ \\
\hline At last examination(week) & & Median \\
\hline
\end{tabular}

Fourty two (42) patients had Doppler (UA, MCA, DV) and biophysical profile scoring performed during the study period. With a mean maternal age $(27.59 \pm 6.03)$ years. Twenty-two $(52.4 \%)$ of women were nulliparous. 
Pre-eclampsia was the most common medical condition, observed in 9 women (21.4\%). Delivery was at a mean of 35.65 weeks. Thirty-one cases $(73.8 \%)$ were delivered by Cesarean section. The mean gestational age at the initial referral was 32.52 weeks gestation. Smoking was present in 4 (9.5\%) patients. Eight patients (19\%) reported past history of IUGR. Mean BMI of the studied population was 28.93. Twenty-two cases (52.4\%) were nulliparous.

Table (2): Distribution of biophysical profile score results.

\begin{tabular}{|c|c|c|}
\hline BPP Score & Normal BPP $28(66.6 \%)$ & Abnormal 14 (33.3\%) \\
\hline 10 & 8 & \\
\hline 8 & 20 & \\
\hline 6 & & 2 \\
\hline 4 & & 8 \\
\hline 2 & & 2 \\
\hline$\overline{\mathbf{0}}$ & & 2 \\
\hline
\end{tabular}

The BPP was > 6 in 28 fetuses (66.6\%). The remaining 14 (33.3\%) fetuses had a score of 6 or less. We related outcome parameters to fetal status as assessed by Doppler ultrasonography and BPP.

Table (3): Distribution of the Biophysical profile regarding APGAR score, Birth weight, EFW, NICU admissions and neonatal outcome.

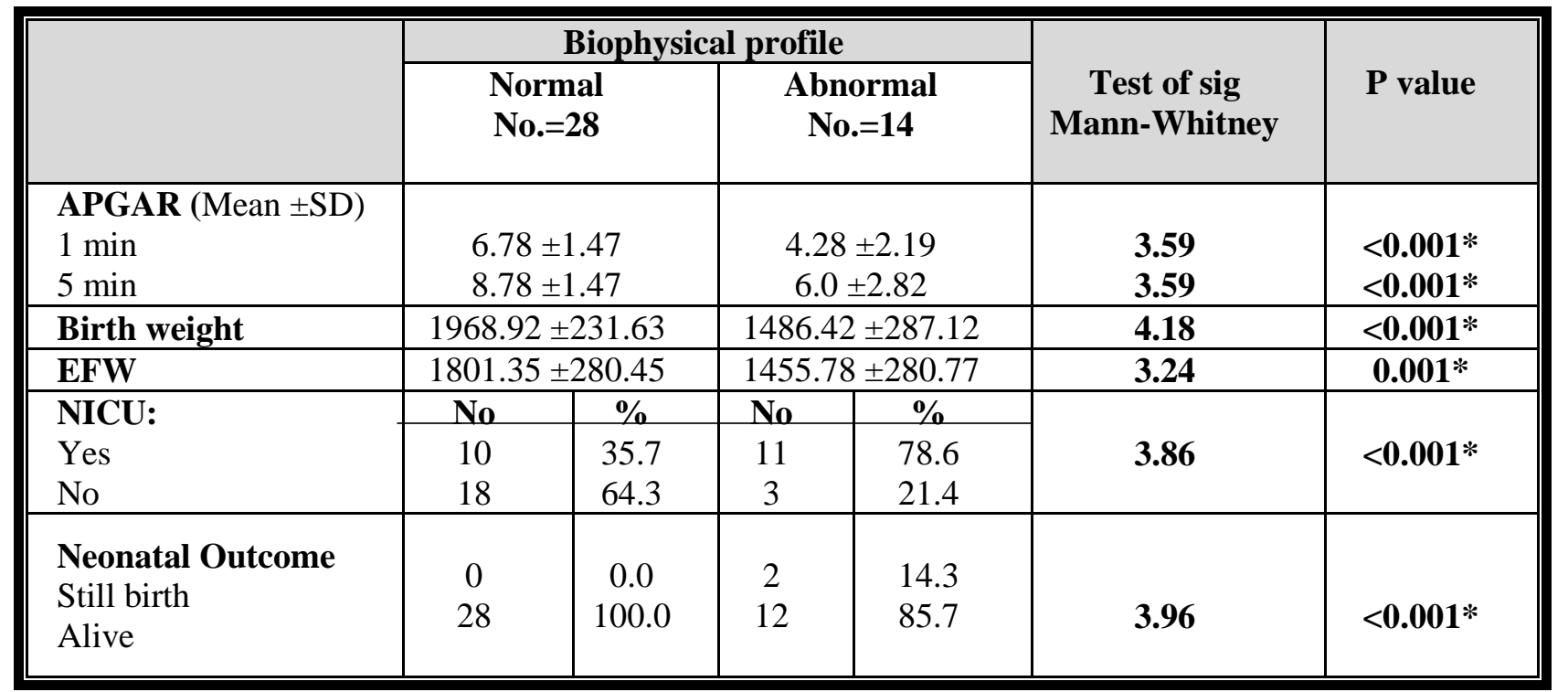

*: significant, EFW: Expected fetal weight, NICU: Neonatal Intensive Care Unit admission

Abnormal BPP is significantly associated with low APGAR score, Birth weight, EFW, increased NICU admissions and stillbirth rate.

Table (4): Distribution of DV and MCP regarding APGAR score, Birth weight, EFW, NICU admissions and neonatal outcome.

\begin{tabular}{|l|l|l|l|l|l|l|}
\hline & & P value & MCP & P value & DV & \\
\hline
\end{tabular}




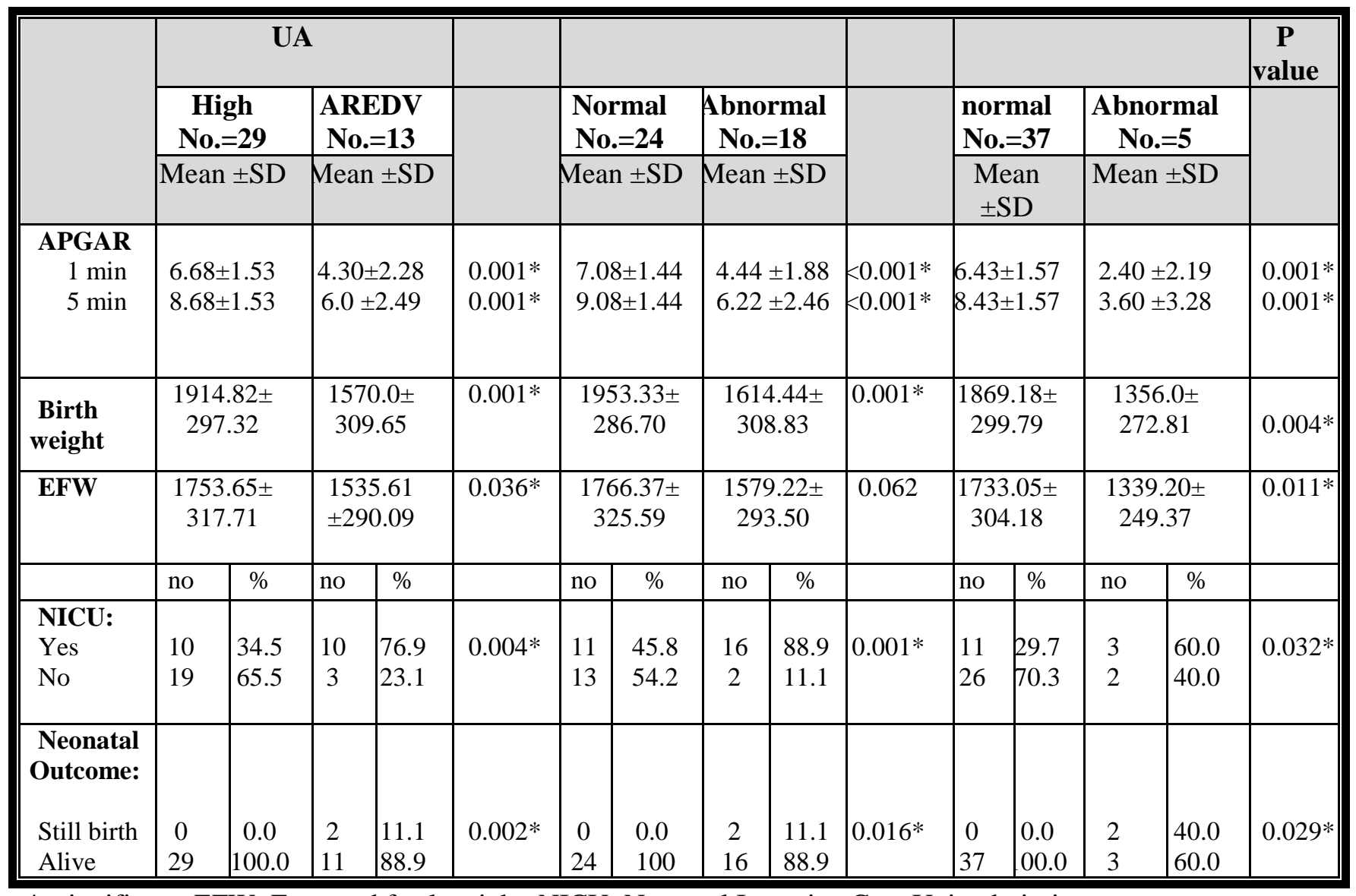

*: significant, EFW: Expected fetal weight, NICU: Neonatal Intensive Care Unit admission Abnormal DV, MCA, UA Doppler are significantly associated with low APGAR score, Birth weight, EFW, increased NICU admissions and stillbirth rate.

Table (5): Multivariate regression analysis for predictor of fetal outcome.

\begin{tabular}{|c|c|c|c|c|c|}
\hline & \multirow[b]{2}{*}{ Wald } & \multirow[b]{2}{*}{$P$ value } & \multirow[b]{2}{*}{ Odds Ratio } & \multicolumn{2}{|c|}{$95 \%$ Confidence Interval } \\
\hline & & & & Lower & Upper \\
\hline Umbilical artery & 0.03 & 0.849 & 1.29 & 0.09 & 17.61 \\
\hline Middle cerebral artery & 5.79 & $0.016^{*}$ & 16.50 & 1.68 & 161.84 \\
\hline Ductus venosus & 3.96 & 0.046 & 0.021 & 0.00 & 0.94 \\
\hline Biophysical profile & 2.88 & 0.089 & 2.79 & 0.85 & 9.11 \\
\hline
\end{tabular}

*: significant

A logistic regression was performed to ascertain the effects of UA, MCP, DV and BPP on the likelihood of admission to NICU. Logistic regression model was statistically significant, $\chi 2=13.55, p=0.022$. The model explained 37.9\% (Nagelkerke R2) of the variance in unsatisfactory knowledge and correctly classified $78.6 \%$ of cases. It was found that abnormal MCA was associated with NICU admission (OR=16.50, CI95\%: 1.68-161.84). UA end diastolic velocity was present in 29 fetuses (69\%), absent in $6(14.28 \%)$ and reversed in $7(16.66 \%)$. Brain sparing was observed in (42.85\%) of fetuses. Abnormal DV Doppler was noticed in 5 fetuses $(11.9 \%)$. 


\section{DISCUSSION}

The search for more accurate surveillance techniques in IUGR fetuses naturally leads to the question of which antenatal testing modality is best Doppler ultrasonography, five-component BPP (including fetal heart rate analysis) or a combination of both modalities? If you are going to investigate this question, you should start analyzing the distribution of test results. If Doppler examination and BPP are coordinating with each other, then a combination of both modalities is clearly less valuable. However, if test results are not in coordination then the combination of both modalities may worth due to the complementarity of the information gained through their combination ${ }^{(\mathbf{1 0})}$.

In our study, a mean maternal age (27.59) years which was near the mean age (29.87) determined by Baschat ${ }^{(10)}$. Twenty-two (52.4\%) of women were nulliparous which was slightly different from the results of the study done by Unterscheider and coworkers (11) where eleven (31.49\%) were nulliparous. Pre-eclampsia was the most common medical condition, observed in 9 women (21.4\%), this finding also observed by Bardakci et al. ${ }^{(\mathbf{1 2})}$ who noted $14(24.8 \%)$ of cases having preeclampsia. Thirty one cases $(73.8 \%)$ were delivered by Cesarean section while in Baschat ${ }^{(\mathbf{1 0})}$ study the mode of delivery was $(80 \%)$ by CS. Arbeille et al. ${ }^{(13)}$ noted that mode of delivery was labor induction in 114 cases $(66.7 \%)$, cesarean delivery in 60 cases $(35.1 \%)$.

The mean gestational age at the initial referral was 32.52 weeks gestation. Delivery was at a mean of 35.65 weeks. This would be in agreement with Bardakci and coworkers ${ }^{(\mathbf{1 2})}$ study where mean GA at delivery was 36.21 weeks. Smoking was present in 4 (9.5\%) patients. Eight patients (19\%) reported past history of IUGR. Mean BMI of the studied population was 28.93. The BPP was > 6 in 28 fetuses $(66.6 \%)$. The remaining $14(33.3 \%)$ fetuses had a score of 6 or less. This was different from Baschat ${ }^{(\mathbf{1 0})}$ where only $46 \%$ of fetuses had normal BPP. In our study, Abnormal BPP was significantly associated with low APGAR score, Birth weight, EFW, increased NICU admissions and stillbirth rate. This was widely agreed by both Baschat $^{(10)}$ and Unterscheider et al. (11) studies.

Also, Abnormal UA, MCA, DV Dopplers were significantly associated with low APGAR score, Birth weight, EFW, increased NICU admissions and

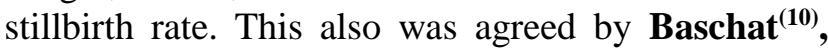
Unterscheider et al. ${ }^{(11)}$ and Bardakci ${ }^{(12)}$ studies. However, we added a multivariate regression analysis for predictors of fetal outcome, and it was found that abnormal MCA was the most associated parameter with NICU admission (OR=16.50, CI95\%: 1.68161.84). That was agreed by Michael Patrick Geary and colleagues ${ }^{(14)}$. Late Doppler changes such as abnormal venous Doppler indices and umbilical venous pulsations are associated with fetal acidosis and the poorest perinatal outcomes ${ }^{(15)}$.

In a parallel but asynchronous way, fetal behavioral responses to placental insufficiency can also be subdivided into early and late. Early changes are predominantly due to maturational delay in the central integration of fetal behaviors. This delayed acquisition of behavioral milestones is only apparent on computerized analysis ${ }^{(\mathbf{1 6})}$.

One of the key points in this study that is different from many previous studies is that we diagnosed the IUGR status of the infant not by antenatal ultrasound assessment only, but also by birth weight measured after birth <10th centile. This method would eliminate possible errors in antenatal birth weight measurement ${ }^{(17)}$. A systematic review of the accuracy of umbilical artery Doppler in a high-risk population to diagnose an IUGR fetus has shown moderate accuracy ${ }^{\mathbf{( 1 8})}$. Doppler ultrasonography and biophysical profile scoring (BPP) are the principal surveillance tools in pregnancies complicated by placental insufficiency and fetal growth restriction (IUGR), but fetal deterioration appears to be independently reflected in these two testing modalities: and their combined use is likely to be complementary ${ }^{(\mathbf{1 9})}$.

Our results are confirming that biometry at the beginning of third trimester (30-34 weeks) was useful for the diagnosis of IUGR fetuses. The sensitivity is improved by adding UA Doppler, MCA PI Doppler velocimetry ${ }^{(20)}$. The possibility of early detection of a risk group for growth deficit can allow us to start preventive measures (fetal monitoring) and to distinguish an IUGR fetus from a constitutional SGA, which grows physiologically throughout pregnancy (21). Middle cerebral artery (MCA) pulsed Doppler has long constituted the clinical standard for the diagnosis of brain redistribution (22). Middle cerebral artery (MCA) PI have been proposed for clinical detection of brain redistribution in growth-restricted fetuses ${ }^{(23)}$.

In this cohort abnormal venous Doppler indices preceded an abnormal BPP in the majority of cases. In the study presented here we have related arterial and venous Doppler ultrasound examination to a more general IUGR population that was preselected by small fetal size associated with evidence of abnormal placentation. Our results suggest that even when DV Doppler ultrasonography is taken into account, Doppler and BPP results appear to be independent. This provides strong evidence that Doppler examination and BPP are complementary antenatal modalities. Therefore, pursuit of a combined testing approach has merit. In developing such an integrated fetal testing modality detailed appraisal of the relationships between individual testing parameters and critical perinatal outcomes will be of importance. 
The strength points of our study are: 1- Our study studied different and multiple Doppler parameters (UA, MCA, DV) and combined it with Biophysical profile score (BPP) 2- we diagnosed the IUGR status of the infant not by antenatal ultrasound assessment only, but also by birth weight measured after birth $<10^{\text {th }}$ centile. This method would eliminate possible errors in antenatal birth weight measurement. 3- We performed Multivariate regression analysis between all the studied parameters, and it was found that abnormal MCA was the most associated with NICU admission.

\section{CONCLUSION}

We support addition of the MCA as the most sensitive Doppler parameter for the clinical monitoring of these fetuses. However, strong evidence that Doppler examination and BPP are complementary antenatal modalities. Therefore, pursuit of a combined testing approach has merit. In developing such an integrated fetal testing modality detailed appraisal of the relationships between individual testing parameters and critical perinatal outcomes will be of importance.

\section{Key points:}

- Intrauterine Growth Restriction is one the most common causes of perinatal morbidity and mortality worldwide.

- There is no clear evidence regarding the best method of surveillance either Doppler (UA, MCA, DV) or Biophysical profile (BPP).

- Up to our knowledge, no studies did regression analysis between the all different parameters regarding the neonatal outcome.

- We support addition of the MCA as the most sensitive Doppler parameter.

- Doppler examination and BPP are complementary antenatal modalities.

\section{Acknowledgements: No declared.}

Financial support and sponsorship: We have not received any funding from any corporate body or pharmaceutical company.

Conflicts of interest: the authors have no conflicts of interest with regard to the contents of this work.

\section{REFERENCES}

1. McGowan J, Alderdice F, Holmes $\mathrm{V}$ et al. (2011): Early childhood development of late-preterm infants: a systematic review. Pediatrics, 127(6):1111-24.

2. Figueras F, Gardosi J (2011): Intrauterine growth restriction: new concepts in antenatal surveillance, diagnosis, and management. Am J Obstet Gynecol., 204(4):288-300.

3. Ferrazzi E, Bozzo M, Rigano $S$ et al. (2002): Temporal sequence of abnormal Doppler changes in the peripheral and central circulatory systems of the severely growth-restricted fetus. Ultrasound Obstet Gynecol., 19: 140-146.

4. Bilardo C, Stigter R, Ville Y et al. (2001): Monitoring of fetuses with intrauterine growth restriction: a longitudinal study. Ultrasound Obstet Gynecol., 18:564-570.

5. Ribbert L, Visser G, Mulder E et al. (1993): Changes with time in fetal heart rate variation, movement incidences and haemodynamics in intrauterine growth retarded fetuses: a longitudinal approach to the assessment of fetal well being. Early Hum Dev., 31: 195-208.

6. Vintzileos A, Fleming A, Scorza W et al. (1991): Relationship between fetal biophysical activities and umbilical cord blood gas values. Am J Obstet Gynecol., 165: 707-713.

7. Manning F, Snijders R, Harman C et al. (1993): Fetal biophysical profile score. VI. Correlation with antepartum umbilical venous fetal $\mathrm{pH}$. Am J Obstet Gynecol., 169: 755-763.

8. Comas M, Crispi F, Cruz-Martinez R et al. (2011): Tissue Doppler echocardiographic markers of cardiac dysfunction in small-for-gestational age fetuses. Am J Obstet Gynecol., 205(1):57-1.

9. Illa M, Coloma J, Eixarch E et al. (2009): Growth deficit in term small-for-gestational fetuses with normal umbilical artery Doppler is associated with adverse outcome. J Perinat Med., 37(1):48-52.

10. Baschat D (2004): Fetal responses to placental insufficiency: an update. BJOG An Int J Obstet Gynaecol., 111(10):1031-41.

11. Unterscheider J, Daly S, Geary M et al. (2013): Optimizing the definition of intrauterine growth restriction: the multicenter prospective PORTO Study. Am J Obstet Gynecol., 208(4):290-6.

12. Bardakci M, Balci O, Acar A et al. (2015): Comparison of Modified Biophysical Profile and Doppler Ultrasound in Predicting the Perinatal Outcome at or over 36 Weeks of Gestation. Am J Obstet Gynecol., 221(3):283-7.

13. Arbeille $P$, Maulik $D$, Fignon A et al. (1995): Assessment of the fetal PO2 changes by cerebral and umbilical Doppler on lamb fetuses during acute hypoxia. Ultrasound Med Biol., 21(7):861-70.

14. Ferrazzi E, Bozzo M, Rigano S et al. (2002): Temporal sequence of abnormal Doppler changes in the peripheral and central circulatory systems of the severely growth-restricted fetus. Ultrasound Obstet Gynecol., 19(2):140-6.

15. Berkley E, Chauhan S, Abuhamad A (2012): Maternal-Fetal Medicine Publications Committee S, others. Doppler assessment of the fetus with intrauterine growth restriction. Am J Obstet Gynecol., 206(4):300-8.

16. Kennelly M, Farah N, Turner M et al. (2010): Aortic isthmus Doppler velocimetry: role in assessment of preterm fetal growth restriction. Prenat Diagnosis Publ Affil With Int Soc Prenat Diagnosis, 30(5):395-401.

17. Del Rio M, Martinez J, Figueras F et al. (2006): Reference ranges for Doppler parameters of the fetal aortic isthmus during the second half of pregnancy. 
Ultrasound Obstet Gynecol Off J Int Soc Ultrasound Obstet Gynecol., 28(1):71-6.

18. Ebbing C, Rasmussen S, Kiserud T (2007): Middle cerebral artery blood flow velocities and pulsatility index and the cerebroplacental pulsatility ratio: longitudinal reference ranges and terms for serial measurements. Ultrasound Obstet Gynecol Off J Int Soc Ultrasound Obstet Gynecol., 30(3):287-96.

19. Morris R, Malin G, Robson S et al. (2011): Fetal umbilical artery Doppler to predict compromise of fetal/neonatal wellbeing in a high-risk population: systematic review and bivariate meta-analysis. Ultrasound Obstet Gynecol., 37(2):135-42.

20. Savchev S, Figueras F, Cruz-Martinez $\mathbf{R}$ et al. (2012): Estimated weight centile as a predictor of perinatal outcome in small-for-gestational-age pregnancies with normal fetal and maternal Doppler indices. Ultrasound Obstet Gynecol., 39(3):299-303.
21. Ebrashy A, Azmy O, Ibrahim M et al. (2005): Middle cerebral/umbilical artery resistance index ratio as sensitive parameter for fetal well-being and neonatal outcome in patients with preeclampsia: case-control study. Croat Med J., 46(5): 6-9.

22. Cruz-Martinez R, Figueras F, Hernandez-Andrade E et al. (2010): Longitudinal brain perfusion changes in near-term small-for-gestational-age fetuses as measured by spectral Doppler indices or by fractional moving blood volume. Am J Obstet Gynecol., 203(1):42-1.

23. Simanaviciute D, Gudmundsson S (2006): Fetal middle cerebral to uterine artery pulsatility index ratios in normal and pre-eclamptic pregnancies. Ultrasound Obstet Gynecol Off $\mathbf{J}$ Int Soc Ultrasound Obstet Gynecol., 28(6):794-801. 